PROCEEDINGS OF THE

AMERICAN MATHEMATICAL SOCIETY

Volume 127, Number 1, January 1999, Pages 131-137

S 0002-9939(99)04919-9

\title{
UNIVERSAL STATE SPACE EMBEDDABILITY OF JORDAN-BANACH ALGEBRAS
}

\author{
JAN HAMHALTER
}

(Communicated by David R. Larson)

\begin{abstract}
We study extensions of states between projection structures of JB algebras and generalized orthomodular posets. It is shown that projection orthoposet of a JB algebra $A$ admits the universal extension property if and only if the Gleason theorem is valid for $A$. As a consequence we get that any positive Stone algebra-valued measure on projection lattice of a quotient of a JBW algebra without type $I_{2}$ direct summand extends to a positive measure on an arbitrary larger generalized orthomodular lattice.
\end{abstract}

The classical extension theorem of Horn and Tarski [5] says that any probability measure on a Boolean algebra $B$ extends to a probability measure on any larger Boolean algebra $B^{\prime}$ containing $B$. The first "quantum measure theoretic" generalization of this result was given by P. Pták [10] who showed that $B^{\prime}$ can be replaced by any quantum logic $L$ with reasonably rich state space. We say in this case that Boolean algebras have the universal state extension property. In further development it has been proved in [6] that the universal state extension property for projection structures of unital $C^{*}$-algebras is equivalent with the existence of "non-commutative integral" (so called Gleason property). As a consequence we get that projection lattices of von Neumann algebras without type $I_{2}$ direct summand enjoy the universal state extension property. The aim of this note is to extend the above stated results for (not necessarily unital) Jordan Banach algebras and corresponding orthomodular structures not containing a largest element. This generalization is based on extension technique using determinacy of pure states on Jordan algebras. Moreover, general extension theorem for complete vector space valued measures on projections will be proved.

First we recall a few notions and fix the notation. (Our standard references for Jordan algebras, ordered vector spaces, and quantum logics are [7, 1, 11], correspondingly.)

By a Jordan algebra we shall mean a real vector space $A$ equipped with the product (i.e. bilinear form) written as $(a, b) \rightarrow a \circ b$ and satisfying the following properties for all $a, b \in A: a \circ b=b \circ a, a \circ\left(b \circ a^{2}\right)=(a \circ b) \circ a^{2}$. $A$ is said to be unital if it admits a unit with respect to the Jordan product.

In addition, if $A$ is a Banach algebra with respect to the product $\circ$ and if the norm $\|\cdot\|$ satisfies the following conditions for all $a, b \in A$ : $\left\|a^{2}\right\| \leq\left\|a^{2}+b^{2}\right\|$,

Received by the editors May 1, 1997.

1991 Mathematics Subject Classification. Primary 46L70, 46L50, 28B15, 81P10.

Key words and phrases. Jordan algebras, extensions of measures on projections, generalized orthomodular posets, Gleason theorem. 
$\left\|a^{2}\right\|=\|a\|^{2}$, then $A$ is said to be a $J B$ algebra. In the sequel $A$ will always denote a JB algebra. If $A$ is a dual Banach space, then $A$ is called a JBW algebra. An important example of a JB algebra is a Banach algebra of self-adjoint operators acting on a Hilbert space $H$ endowed with a product $a \circ b=1 / 2(a b+b a)$. Such an algebra is called a JC algebra. Any JC algebra which is weakly operator closed is said to be a JW algebra.

We write $A_{1}=\{a \in A ;\|a\| \leq 1\}, A^{+}=\left\{a^{2} ; a \in A\right\}, A_{1}^{+}=A_{1} \cap A^{+}$. For $a \in A$ mappings $T_{a}, U_{a}: A \rightarrow A$ are defined by letting $T_{a}(b)=a \circ b, U_{a}(b)=$ $2 a \circ(a \circ b)-a^{2} \circ b$. It is well known that $U_{a}\left(A^{+}\right) \subset A^{+}$. Two elements $a, b$ in $A$ are said to be operator commuting if $T_{a} T_{b}=T_{b} T_{a}$. The center of $A$ is the set of all elements operator commuting with whole algebra $A$. A subspace $I$ of $A$ is called a Jordan ideal if $T_{a}(A) \subset I$ for all $a \in I$. Denote by $A^{*}$ the dual space of $A$. An element $f \in A^{*}$ is said to be positive if $f\left(A^{+}\right) \subset[0, \infty)$. The state space $S(A)$ and the quasi state space $Q(A)$ of $A$ is defined as the set $S(A)=\left\{f \in A^{*} \mid\|f\|=1, f \geq 0\right\}$ and $Q(A)=\left\{f \in A^{*} \mid\|f\| \leq 1, f \geq 0\right\}$, respectively. The extreme points of $S(A)$ are called pure states. Every state of $A$ can be canonically extended to a normal state of $A^{* *}$. We will always denote this extension by the same letter. For any pure state $\varrho$ of $A$ there is a unique minimal projection, $s(\varrho)$, in $A^{* *}$ such that $\varrho(s(\varrho))=1$. Denote by $\mathcal{L}_{\varrho}=\left\{a \in A \mid \varrho\left(a^{2}\right)=0\right\}$ the left kernel of $\varrho$. Then $\mathcal{L}_{\varrho}$ is a subalgebra and $\mathcal{L}_{\varrho}=U_{(1-s(\varrho))}\left(A^{* *}\right) \cap A,(\operatorname{Ker} \varrho)^{+}=\mathcal{L}_{\varrho}^{+}$. The representation $\pi_{\varrho}$ associated with $\varrho$ is defined as a Jordan homomorphism $\pi_{\varrho}: A \rightarrow A^{* *}: a \rightarrow c(\varrho) \circ a$, where $c(\varrho)$ is the smallest (i.e. minimal) projection in the center of $A^{* *}$ which covers $s(\varrho)$.

Let $P(A)=\left\{p \mid p^{2}=p\right\}$ be the set of all projections of a (not necessarily unital) JB algebra $A$. When we endow $P(A)$ with partial ordering inherited from $A$ and consider partial orthocomplementation $q \rightarrow p-q$ defined on each interval $[0, p]$, then $P(A)$ becomes an orthomodular structure known as the generalized orthomodular poset. We call $P(A)$ the projection orthoposet of $A$.

Definition (see [8]). Let $(L, \leq)$ be a partially ordered set with a least element 0 , such that every interval $[0, a]$ in $L$ is equipped with an order-reversing, involutive unary operation $x \rightarrow x^{\perp_{a}}$. L is said to be a generalized orthomodular poset if the following conditions are satisfied for all $a, b \in L$ :

(i) If $a, b \leq c$, and $a \leq b^{\perp_{c}}$, then the supremum $a \vee b$ exists in $L$;

(ii) If $a \leq b$, then $b=a \vee\left(b \wedge a^{\perp_{b}}\right)$ (orthomodular law);

(iii) If $a \leq b \leq c$, then $a^{\perp_{b}}=a^{\perp_{c}} \wedge b$;

(iv) If $a \perp b, c \perp a$, and $c \perp b$, then $c \perp a \vee b$. (We write $a \perp b$ if the supremum $a \vee b$ exists in $A$ and $a \leq b^{\perp_{a \vee b}}$. Elements $a, b$ are said to be orthogonal in this case.)

In what follows let $L$ denote a generalized orthomodular poset. We say that elements $a, b \in L$ commute if $a=b \vee c, b=b \vee d$, where $b \perp c, b \perp d$. A generalized orthomodular poset is said to be Boolean if it consists of mutually commuting elements.

$L$ is a suborthoposet of a generalized orthomodular poset $K$ (in the category of generalized orthomodular posets) if $L \subset K$ and if the ordering, the least element, the orthocomplementation operation and the formation of suprema of orthogonal elements coincide for $K$ and $L$ on every interval $[0, a], a \in L$.

A bounded real-valued function $m$ on $L$ is said to be a measure if $m(a \vee b)=$ $m(a)+m(b)$, whenever $a \perp b$. By a state $s$ on $L$ (probability measure) we mean a measure $s: L \rightarrow[0,1]$ satisfying $\sup _{a \in L} s(a)=1$. The set of all states will 
be denoted by $S(L)$. The quasi-state space $Q(L)$ of $L$ is the set of all measures on $L$ with values in the interval $[0,1]$ endowed with the topology $\tau$ of pointwise convergence on elements of $L$. Thus $(Q(L), \tau)$ is a compact Hausdorff space which is a convex set in $R^{L}$. An element of $Q(L)$ is called pure if it is an extreme point of $Q(L) . L$ is said to have a unital set of states if for every non-zero $a \in L$ there is a state $s$ of $L$ such that $s(a)=1$.

We say that $L$ has the universal state extension property if the following condition is satisfied. Let $K$ be a generalized orthomodular poset with a unital set of states containing $L$ as a suborthoposet. Then every state of $L$ extends to a state of $K$. It can be proved by an easy modification of [10] that every Boolean generalized orthoposet admits the universal state extension property. In case of projection orthoposets the following important extension property is often considered. A JB algebra has $A$ the Gleason property if every state of $P(A)$ extends to a state of $A$. According to the generalized Gleason theorem for Jordan algebras [2, 3] any JBW algebra not containing type $I_{2}$ direct summand has the Gleason property. Further, any JB algebra whose projection orthoposet has the universal state extension property has the Gleason property. This fact is due to the observation that any JB algebra can be embedded into a JBW algebra not containing a type $I_{2}$ direct summand. It has been proved in [6] that, perhaps surprisingly, the Gleason property of unital $\mathrm{C}^{*}$-algebras with sufficiently many projections already implies the universal state extension property. We are now going to generalize this result for non-unital JB algebras. Our extension technique is based on the existence of a determining element shown in the following Proposition. Its proof simplifies considerably in a unital case.

Proposition. For any pure state $\varrho$ on a separable JB algebra $A$ there is an element $x \in A_{1}^{+}$such that $\varrho(x)=1$ and $\varrho$ is the only pure state of $A$ taking value 1 at $x$.

Proof. First we prove that any pure state on a JB algebra is a norm attaining functional. For this let us examine the representation $\pi_{\varrho}$. By the classification of type I JBW factors either $A^{* *} c(\varrho)$ is reflexive, in which case $\pi_{\varrho}(A)=A^{* *} c(\varrho)$, or $A^{* *} c(\varrho)$ may be realized as an irreducible and reversible JW subalgebra of $B(H)$, for some Hilbert space, $H$. In that case $\pi_{\varrho}(A)$ acts irreducibly on $H$ also.

In the first case there is an element $b \in A^{+}$with norm at least 1 such that $\pi_{\varrho}(b)$ is a unit $u$ for $\pi_{\varrho}(A)$. Let $0 \leq f \leq 1$ be a continuous function on the interval $[0,\|b\|]$ with $f(0)=0$ and $f(1)=1$. Then

$$
\pi_{\varrho}(f(b))=f\left(\pi_{\varrho}(b)\right)=f(u)=u .
$$

In the second case take a unit vector $\omega \in s(\varrho)(H)$. It is easy to verify that

$$
\left(\pi_{\varrho}(x) \omega, \omega\right)=\varrho(x) \text { for any } x \in A .
$$

Since $s(\varrho) \omega=\omega$ the transitivity theorem [12] guarantees the existence of a positive element $b \in A$ with $\pi_{\varrho}(b) \omega=\omega$. By the above argument we can modify $b$ to be a positive norm one element.

In both cases we have an element, say $a$, such that $0 \leq a \leq 1$ and $\varrho(a)=1$. Since $\mathcal{L}_{\varrho}$ is a separable JB algebra it contains a strictly positive contraction $y$. Now $x=a-U_{a}(y)$ satisfies the statement of the Proposition. Indeed, $0 \leq x \leq 1$. As $\varrho(a)=1$, we have $\varrho\left(U_{a}(y)\right)=\varrho(y)=0$ by the Cauchy-Schwarz inequality. Hence, $\varrho(x)=1$. If $\varphi$ is another pure state with $\varphi(x)=1$, then $\varphi(a)=1, \varphi\left(U_{a}(y)\right)=0$. So, $\varphi(y)=0$. But then $\varphi \mid \mathcal{L}_{\varrho}=0$, and, in turn, $\varphi(1-s(\varrho))=0$ by normality of $\varphi$ 
on $A^{* *}$. Equivalently, $\varphi(s(\varrho))=1$ and so $\varphi=\varrho$ by uniqueness of the support of a pure state. The proof is completed.

Theorem 1. Let $A$ be a JB algebra such that every element $x$ of $A$ is contained in the closed linear span of some family of mutually operator commuting projections. Then A has the Gleason property if and only if $P(A)$ has the universal state extension property.

Proof. Assume that $A$ has the Gleason property and $L$ is a generalized orthomodular poset with a unital set of states containing $P(A)$ as a substructure. Given a state $\varphi$ of $A$ we will seek an element of $Q(L)$ extending $\varphi \mid P(A)$.

Substantial part of the proof consists in proving that $\varphi \mid P(M)$ extends to an element of $Q(L)$ provided that $M$ is a separable subalgebra of $A$. At the first stage we will prove it under the assumption that $\varphi \mid M$ is a pure state. According to the Proposition there is a determining element $x \in M$ for the state $\varphi \mid M$. Let $B$ be an associative JB subalgebra of $A$ which contains $x$ in a norm closed linear span of its projections. Since $P(B)$ is a Boolean generalized orthomodular poset, $P(B)$ enjoys the universal state extension property and $\varphi \mid P(B)$ extends to $\bar{\varphi} \in Q(L)$. Let $\hat{\varphi}$ be a linear extension of $\bar{\varphi} \mid P(A)$. Then $\hat{\varphi}(x)=\varphi(x)$ and, in turn, $\hat{\varphi}=\varphi$ on $M$. Therefore $\bar{\varphi}$ is an extension of $\varphi \mid P(M)$.

Let us now consider the general case of $\varphi \mid M$ being an arbitrary element of $Q(M)$. Since every extreme point of the compact convex set $Q(M)$ is either a pure state of $M$ or zero, the Krein-Milman theorem says that $\varphi \mid M$ is a weak*-limit point of a net $\left(\psi_{\alpha}\right)$, where each $\psi_{\alpha}$ is a linear combination of pure states of $M$ whose coefficients are from interval $[0,1]$. Every state $\psi_{\alpha} \mid P(M)$ extends to a state $\widetilde{\psi}_{\alpha}$ of $L$. Employing now compactness of $Q(L)$ we may assume (passing to a subnet if necessary) that $\widetilde{\psi}_{\alpha} \rightarrow \widetilde{\psi}$. Then $\widetilde{\psi}$ is the desired extension.

Now let $p_{1}, \ldots, p_{n}$ be projections in $A$. Considering a JB algebra $A\left[p_{1}, \ldots, p_{n}\right]$ generated by elements $p_{1}, \ldots, p_{n}$, we can extend $\varphi \mid P\left(A\left[p_{1}, \ldots, p_{n}\right]\right)$ to a measure in $Q(L)$. In other words, for each finite subset $S \subset P(A)$, the set $F_{S}=\{\varrho \in$ $Q(L) ; \varrho|S=\varphi| S\}$ is a nonempty (compact) set in $Q(L)$. As $F_{\cup S_{i}}=\bigcap F_{S_{i}}$ for any finite collection $S_{1}, \ldots, S_{n}$ of finite subsets of $P(A)$, it follows immediately that the system $\left(F_{S}\right)_{S \in \Gamma}$, where $\Gamma$ is a collection of all finite subsets of $P(A)$, has the finite intersection property. The set $Q(L)$ being compact, the intersection $\bigcap_{S \in \Gamma} F_{S}$ has to be non-void and any element $\widetilde{\varphi} \in \bigcap_{S \in \Gamma} F_{S}$ serves as a state of $L$ extending $\varphi \mid P(A)$. The proof is complete.

Corollary. Let I be a norm closed Jordan ideal in a JBW algebra M. Suppose that $M$ does not contain type $I_{2}$ direct summand. Then the quotient structure $P(M / I)$ enjoys the universal state extension property.

Proof. By $[2,3] M$ has the Gleason property and so does the quotient $M / I$. Take $x \in M / I$. Denote by $\pi$ the canonical quotient map. Then $x=\pi(y)$ for some $y \in M$. Let $B$ be an associative JBW subalgebra of $M$ generated by $y$. Then $x$ is in the norm closed span of projections in $\pi(B)$. Thus the quotient $M / I$ meets all assumptions of Theorem 1 and the proof is complete.

Restating the Corollary we see that any positive measure on the projection lattice of a JBW algebra, and von Neumann algebra, usually extends to a positive measure on an arbitrary larger generalized orthomodular structure. (Recall that we require only projection lattice to live in some subinterval of a superstructure.) In the 
conclusion of this note we show that this extension theorem remains valid even for more general vector measures having values in vector lattices. Since the proof is based on the techniques of ordered vector spaces we first introduce ordered linear space naturally associated with a given orthomodular poset.

Let $L$ be a generalized orthomodular poset. We denote by $A(L)$ ("linearization" of $L$ ) the space of all bounded affine functions on the quasi-state space $Q(L)$ vanishing at 0 . Endowed with the usual arithmetic operations, supremum norm, and pointwise order, $(A(L), \leq)$ becomes an ordered Banach space. It can be easily verified that the function $1_{L}$ defined by $1_{L}(s)=\sup _{a \in L} s(a), s \in Q(L)$, is an affine function on $Q(L)$ on condition that $L$ is a lattice (or at least upwards directed ordered set). In that case $\left(A(L), \leq, 1_{L}\right)$ forms an order-unit Banach space. The structure $L$ can be mapped into $A(L)$ via evaluation mapping, $e_{L}$, given by

$$
\left[e_{L}(a)\right](s)=s(a), \text { for all } s \in Q(L) \text { and } a \in L .
$$

Then, of course, $e_{L}(a \vee b)=e_{L}(a)+e_{L}(b)$ whenever $a \perp b$. If the set $Q(L)$ is order determining in the sense that $a \leq b \Longleftrightarrow s(a) \leq s(b)$ for all $s \in Q(L)$, then $e_{L}$ is injective and order preserving.

Suppose now that $L$ has the universal state extension property and that $K$ is a generalized orthomodular lattice with unital set of states containing $L$. It can be proved in the same way as in the unital case [6, Lemma 3] that the mapping $i: A(L) \rightarrow A(K), f \rightarrow i(f)$, defined by

$$
i(f)(s)=f(s \mid L), \quad s \in Q(K),
$$

is a linear isometry preserving the order. Thus, $A(L)$ can be viewed as a subspace of $A(K)$.

Finally, recall that an order unit-space $\left(S, \leq, 1_{S}\right)$ is called a complete vector lattice if $S$ is a complete lattice with respect to $\leq$. Every complete vector lattice is isomorphic to some Stone algebra, $C(X)$, of continuous functions on some extremely disconnected compact Hausdorff space $X$. By a positive $S$-valued measure on a generalized orthomodular poset $L$ we mean a mapping $\mu$ of $L$ into a positive part of $S$ such that $\mu(a \vee b)=\mu(a)+\mu(b)$ whenever $a \perp b$, and $\mu(L)$ is bounded in $S$.

Theorem 2. Let $M$ be a quotient of a JBW algebra $W$, where $W$ does not contain a type $I_{2}$ direct summand. Let $\mu$ be a positive $S$-valued measure on $P(M)$, where $\left(S, \leq, 1_{S}\right)$ is a complete vector lattice such that $\mu\left(1_{M}\right)=1_{S}$. Then $\mu$ extends to a positive $S$-valued measure on any larger generalized orthomodular lattice $K$ with a unital set of states containing $P(M)$.

Proof. According to the Corollary $P(M)$ enjoys the universal state extension property and so the mapping $i: A(P(M)) \rightarrow A(K)$ defined above is an embedding in the category of ordered vector spaces. Denote by $(V, v)$ the order-unit space $\left(i(A(P(M)), \leq, v)\right.$, where $v=\left(i \circ e_{P(M)}\right)\left(1_{M}\right)$. By the Gleason Property we can identify state spaces $S(P(M))$ and $S(M)$ and so $A(P(M))$ is isomorphic to the second dual $M^{* *}$. By [4] (easily modified for quotients) the $S$-valued measure $\mu$ extends to a positive unital linear mapping $\hat{\mu}$ of $M$ into $S$. By the extension property of vector lattices proved in [9] we can further extend $\hat{\mu}$ to a linear mapping $\tilde{\mu}: M^{* *} \rightarrow S$ with $\|\tilde{\mu}\|=\|\hat{\mu}\|$. Since $\|\tilde{\mu}\|=\tilde{\mu}\left(1_{M}\right)$ we have by [1, Proposition II 1.3 , p. 69$]$ that $\tilde{\mu}$ is positive. Summing it up, $\mu$ induces a positive linear mapping $F: V \rightarrow S$ such that

$$
\mu=F \circ i \circ e_{P(M)} .
$$


Since $K$ is a lattice, the function $1_{K}$ is an order unit for $A(K)$. We can define a linear mapping $\bar{F}: V+R 1_{K} \rightarrow S$ extending $F$ and satisfying $\bar{F}\left(1_{K}\right)=1_{S}$. Let us show that $\bar{F}$ is also positive. Suppose that $x+\lambda 1_{K} \geq 0$ for $x \in V$, and $\lambda \in R$. Take a state $s$ of a lattice $K$ satisfying $v(s)=1$. Then

$$
\left(x+\lambda 1_{K}\right)(s)=x(s)+\lambda \geq 0, \text { and, in turn, }(x+\lambda v)(s)=x(s)+\lambda \geq 0 .
$$

Thus, the preimage $i^{-1}(x+\lambda v)$ is positive and so is $x+\lambda v$. Hence,

$$
\bar{F}\left(x+\lambda 1_{K}\right)=F(x)+\lambda 1_{S}=F(x+\lambda v) \geq 0
$$

due to positivity of $F$. It follows that $\|\bar{F}\|=1$. Using extension property of $\left(S, 1_{S}\right)$ we can further extend $\bar{F}$ to a continuous linear mapping $\widetilde{F}: A(K) \rightarrow S$ such that $\|\widetilde{F}\|=\|\bar{F}\|=1$. Since $\widetilde{F}\left(1_{K}\right)=\bar{F}(v)=1_{S}$ we see that $\widetilde{F}$ is positive. It is now clear that the $S$-valued function $\hat{\mu}$ on $K$ given by

$$
\hat{\mu}=\widetilde{F} \circ e_{K}
$$

is an $S$-valued measure. Using the fact that $e_{K} \mid P(M)=i \circ e_{P(M)}$ we get for $p \in P(M)$ that

$$
\hat{\mu}(p)=\left(\widetilde{F} \circ e_{K}\right)(p)=\left(\widetilde{F} \circ i \circ e_{P(M)}\right)(p)=\left(F \circ i \circ e_{P(M)}\right)(p)=\mu(p) .
$$

Hence, $\hat{\mu}$ is the desired extension and the proof is complete.

Let us remark at the end of this note that Theorem 2 also holds for general (not necessarily positive) $S$-valued measures. This can be justified by inspection of the proof and recent results of L. J. Bunce and J. D. M. Wright on linear extensions of signed measures on von Neumann projection lattices.

Theorem 2 also has the following consequence for the case of Boolean algebras studied in $[5,10]$. Let us consider the situation when a complete Boolean algebra $B$ lives in a generalized orthomodular lattice $K$ which has a unital set of states. Then the identity map on $B$ can be considered as a positive measure on $B$ with values in the Stone algebra $C(X)$ of $B$. Since $C(X)$ is a complete vector lattice, Theorem 2 applies to give a positive measure $E: K \rightarrow C(X)$ which restricts to the identity on $B$. Since $E$ is induced (after obvious identification) by a norm one projection of $A(K)$ onto $A(B), E$ can be viewed as a lattice-theoretic counterpart of conditional expectation in operator algebras.

\section{ACKNOWLEDGEMENTS}

The author would like to express his gratitude to the Alexander von Humboldt Foundation, Bonn, for the support of his research the results of which are contained in this paper. He also thanks Prof. D. Kölzow who acted as scientific host during the author's stay at the Mathematical Institute of the University of Erlangen-Nürnberg for his hospitality, helpful discussions and encouragement. Finally, it is a pleasure to acknowledge the support of the Grant Agency of the Czech Republic (Grant No. 201/96/0117).

\section{REFERENCES}

[1] E. M. Alfsen, Compact Convex Sets and Boundary Integral, Springer-Verlag New York, Heidelberg, Berlin (1971). MR 56:3615

[2] L. J. Bunce and J. D. M. Wright, Continuity and linear extensions of quantum measures on Jordan algebras, Math. Scand. 64, (1989), 300-306. MR 91f:46096 
[3] L. J. Bunce and J. D. M. Wright, Quantum measures and states on Jordan algebras, Commun. Math. Phys., 98, (1985), 187-202. MR 86k:46101

[4] L. J. Bunce and J. D. M Wright, On Dye's theorem for Jordan operator algebras, Expositiones Mathematicae, 11, (1993), 91-95. MR 94b:46097

[5] A. Horn and A. Tarski, Measures in Boolean algebras, Trans. Amer. Math. Soc. 64, (1948), 467-497. MR 10:518h

[6] J. Hamhalter, Gleason property and extensions of states on projection logics, Bull. London Math. Soc. 26 (1994), 367-372. MR 95m:46103

[7] H. Hanche-Olsen and E. Stormer, Jordan Operator Algebras, Pitman Publishing, Boston, London, Melbourne, (1984). MR 86a:46092

[8] A. Mayet-Ippolito, Generalized orthomodular posets, Demonstration Mathematica, Vol. XXIV, No. 1-2, (1991), 263-274. MR 92j:06011

[9] L. Nachbin, A theorem of the Hahn-Banach type for linear transformations Trans. Amer. Math. Soc., 68, (1950), 265-274. MR 11:369a

[10] P. Pták, On extensions of states on logics, Bull. Polish Acad. Sci. Math. 33, (1985), 493-497. MR 87e:03158

[11] P. Pták and S. Pulmannová, Orthomodular Structures as Quantum Logics, Academic Publishers, Dordrecht, (Boston), London, (1991). MR 94d:81018b

[12] E. Stormer, Irreducible Jordan algebras of self-adjoint operators, Trans. Amer. Math. Soc., 130, (1968), 153-166. MR 36:700

Permanent address: Czech Technical University-El.Eng., Department of Mathematics, 16627 Prague 6, Czech Republic

E-mail address: hamhalte@math.feld.cvut.cz

Temporary address: Mathematical Institute, University of Erlangen-Nürnberg, Bismarkstrasse 1 1/2, D 91054 Erlangen, Germany

E-mail address: hamhal@mi.uni-erlangen.de 